\title{
Análisis del conocimiento sobre la administración de fármacos
}

\author{
Juan M. Carmona ${ }^{1}$, Ana M. Baena ${ }^{2}$, Ana C. Berral ${ }^{3}$, Quintiliano \\ Sotelo $^{4}$, Beatriz Recio ${ }^{1}$ e Irene Recio ${ }^{1}$ \\ ${ }^{1}$ Socoservis S.L. (España); ${ }^{2}$ Centro de Minusválidos Psíquicos Profundos \\ de Alcolea (España); ${ }^{3}$ Empresa Pública Hospitalaria Alto \\ Guadalquivir (España); ${ }^{4}$ ASMEDICORA S.L. (España)
}

\begin{abstract}
El objetivo de este estudio fue conocer el conocimiento de los profesionales sanitarios del Hospital de Montilla sobre la administración de medicamentos en urgencias. Material: Estudio descriptivo de corte transversal realizado en el Hospital de Montilla (Córdoba). Para ello se distribuyó un cuestionario a médicos y enfermeros mediante un muestreo aleatorio durante el mes de diciembre de 2014. Se utilizó una encuesta de Machado de Azevedo et al. (2012) compuesta de 9 ítems para la valoración de los conocimientos sobre la administración de medicamentos. Resultados: la muestra estuvo compuesta por un $59.1 \%$ de médicos y un $40.9 \%$ de enfermeros con una edad media de 38.05 ( $D S \pm 8.981$ ). Con respecto a la situación los encuestados, un $72.7 \%$ habían recibido formación previa. Con respecto a su propia autoevaluación, el $72.7 \%$ considera tener unos conocimientos satisfactorios sobre la administración de fármacos. El $90.9 \%$ conoce la existencia de protocolo sobre la administración de fármacos en su unidad. Discusión: Aunque el conocimiento de la administración de fármacos es aceptable para el personal sanitario, hay diferencias entre los dos colectivos analizados. Por ello, sería ideal realizar una adecuada formación y reciclaje del personal para conseguir un óptimo conocimiento y, de esta forma, mejorar la atención sanitaria.
\end{abstract}

Palabras clave: Administración de fármacos, farmacología, urgencias, conocimientos enfermeros.

Analysis of knowledge on drug delivery. The objective of this study was to determine the knowledge of health professionals Hospital of Montilla on the administration of drugs in emergencies. Material: cross-sectional descriptive study conducted at the Hospital of Montilla (Córdoba). A questionnaire to doctors and nurses were distributed by random sampling during the month of December 2014. A survey of Machado de Azevedo et al. (2012) that consists of 9 items for the assessment of knowledge on medication administration was used. Results: The sample was composed of $59.1 \%$ of physicians and $40.9 \%$ of nurses with an average age of 38.05 ( $S D \pm 8.981)$. Regarding the situation of respondents, $72.7 \%$ had received prior training. Regarding their own self-assessment, $72.7 \%$ considered to have a satisfactory knowledge of drug administration. $90.9 \%$ of respondents known to exist protocols on the administration of drugs in his unit. Discussion: Although the knowledge of drug delivery is acceptable for health workers, there are differences between the two analyzed collectives. Therefore, it would be ideal to perform adequate training and retraining of staff for optimal knowledge and, in this way, improve health care.

Keywords: Drug delivery, pharmacology, emergency, nursing knowledge.

Correspondencia: Juan Manuel Carmona Torres. Socoservis S.L. C/ Balandro N/19, 11379 Palmones (Los Barrios), Cádiz (España). Email: juanma061988@gmail.com 
Uno de los principios del Juramento Hipocrático "primero no dañar” ha sido una guía para los profesionales de la salud. No obstante, debido a los errores y eventos adversos el personal sanitario puede provocar daño sin intencionalidad alguna a los usuarios durante el ejercicio de su praxis diaria ( $\mathrm{O}^{\prime}$ Leary, 2000).

Uno de los errores más frecuentes en el ejercicio de la práctica diaria de enfermería es la administración errónea de medicación. Según Díaz-Navarlaz y Seguí-Gómez (2006), la creciente complejidad de los tratamientos y los sistemas de administración en que intervienen diversos profesionales facilita que se cometan dichos errores. A nivel internacional se ha establecido que los errores en medicación intravenosa presentan una incidencia que oscila entre el 18\% y el $81 \%$ (Abellón et al., 2013). El amplio margen de diferencia puede deberse a diferencias en el ámbito de estudio y la metodología utilizada en las diferentes publicaciones (Pastó-Cardona et al., 2009). A nivel nacional, según el estudio realizado por Díaz-Navarlaz y Seguí-Gómez (2006), un 64\% de los enfermeros encuestados reconocía haber cometido alguna vez un error de medicación.

Para que esto no ocurra, existe una regla nemotécnica, denominada "los nueve correctos", que aunque no garantiza que no se produzcan errores en la administración, si es cierto que puede prevenir los errores de manera significativa y puede mejorar la seguridad y la calidad de la atención prestada al paciente durante el proceso de administración de medicamentos (Ministerio da Saúde y ANVISA, 2013). Los nueve correctos para administración de medicamentos son (Elliott y Liu, 2010):

-Paciente correcto.

-Medicamento correcto.

-Vía correcta.

-Horario correcto.

-Dosis correcta.

-Registro correcto.

-Acción correcta.

-Forma correcta.

-Respuesta correcta.

El personal de enfermería es legalmente responsable tanto de la correcta administración de los fármacos como de la notificación de consecuencias adversas a los mismos (Rabadán et al., 2002). Por eso, los enfermeros deben de evitar los errores en la administración de medicamentos, ya que la enfermería se sitúa al final de la cadena del proceso de administración de medicación, donde, además, se pueden acumular errores por prescripción y dispensación incorrectas (Low y Belcher, 2002). Los facultativos, por su parte, pueden evitar los errores realizando una correcta prescripción.

El Servicio de Cuidados Críticos y Urgencias es un área de alto riesgo para que ocurran eventos indeseables, debido al alto número de profesionales que trabajan en 
el servicio, a los turnos rotatorios que conlleva el relevo del cuidado de una persona, la gran demanda de pacientes con diversos niveles de gravedad, la deficiencia cuantitativa y cualitativa de los recursos humanos y materiales, la sobrecarga de trabajo y el estrés del profesional (de Oliveira, de Camargo, y Cassiani, 2005; de Souza, da Silva, y Nori, 2007). Además, el colectivo de personas mayores es uno de los más vulnerables a padecer estos eventos indeseables ya que son un colectivo con alto consumo de medicamentos.

Por ello, la administración de medicamentos en este servicio exige un cuidado continuo y unos conocimientos específicos, puesto que cualquier error en el desempeño profesional puede provocar consecuencias graves e irreversibles, como por ejemplo: reacciones adversas, reacciones alérgicas y errores en la administración de medicamentos (da Silva et al., 2009; de Oliveira et al., 2005; de Souza et al., 2007).

Los estudios más recientes como el de da Silva, da Conceição, y Leite (2008) o el de Carvalho y Bortoli (2001) ponen de manifiesto que los errores en la administración de medicamentos suponen consecuencias negativas en el Servicio Sanitario repercutiendo negativamente en los indicadores de asistencia y en los resultados institucionales (morbilidad, mortalidad, prolongación de estancias hospitalarias, reingresos, aumento del gasto sanitario, etc.).

Por tanto, la formación continua del profesional sanitario del Servicio de Cuidados Críticos y Urgencias es la única medida eficaz para garantizar la calidad en la atención, ya que, gracias a la formación, el profesional sanitario alcanza las competencias necesarias para prestar unos cuidados de calidad, seguros y libre de riesgos (da Silva et al., 2008).

\section{Objetivo}

El objetivo de este presente estudio es cuantificar el conocimiento de los profesionales sanitarios del Hospital de Montilla (Córdoba) acerca de la administración de medicamentos en urgencias y emergencias.

\section{MÉTODO}

\section{Participantes}

Se trata de un estudio descriptivo de corte transversal realizado en el Hospital de Montilla (Córdoba), perteneciente a la Agencia Sanitaria Hospitalaria Alto Guadalquivir (ASHAG). La referencia utilizada para el cálculo muestral fue un estudio realizado por et Machado de Azevedo et al. (2012) en el que el 41.5\% de los enfermeros no contestaron correctamente la mitad de los ítems. El tamaño muestral se calculó con el programa GRANMO; una muestra aleatoria de 22 individuos es suficiente para estimar, con una confianza del $95 \%$ y una precisión de $+/-20$ unidades porcentuales, un 
porcentaje poblacional que se prevé que sea alrededor del $41.5 \%$. El porcentaje de reposiciones necesaria se ha previsto que será del $5 \%$.

La muestra estuvo compuesta por un total de 22 profesionales de la salud, siendo el $59.1 \%$ médicos y un $40.9 \%$ de enfermeros con una edad media de 38.05 [Desviación Típica $(D S) \pm 8.981]$ y una media de tiempo trabajado en el Servicio actual de $8.68(D S \pm 5.103)$.

\section{Instrumentos}

Como instrumento se ha utilizado una encuesta de Machado de Azevedo et al. (2012) compuesta de 9 ítems para la valoración de los conocimientos sobre la administración de medicamentos y otra serie de preguntas orientadas a analizar la situación de los profesionales encuestados y su propia autovaloración de conocimientos sobre farmacología.

\section{Procedimiento}

En primer lugar, se distribuyó el cuestionario de conocimientos sobre la administración de medicamentos a 22 enfermeros y médicos del Hospital de Montilla (Córdoba) mediante muestreo aleatorio. Una vez rellenados los cuestionarios se realizó el análisis estadístico. Este estudio, consiste en un estudio descriptivo de corte transversal.

\section{Análisis de datos}

Para el análisis estadístico de los datos se realizó con el programa PASW Statistic 18 (antes SPSS) y consistió en:

-Análisis descriptivo para las variables cualitativas mediante el cálculo de recuentos (n) y proporciones (\%); y para las variables cuantitativas mediante el cálculo de medias aritméticas $(M)$ y desviaciones típicas $(D S)$, medianas (me) y rangos intercuartílicos (RIC), valores mínimos (Min) y máximos (Max).

-Comparación de proporciones de las variables categóricas mediante pruebas chi-cuadrado para tablas de contingencia; en el caso de tablas 2x2 se utilizó el estadístico chi-cuadrado con corrección de Yates, y cuando alguna frecuencia esperada fue $\leq 5$ se aplicó la prueba exacta de Fisher.

Todos los contrastes de hipótesis fueron bilaterales. Y en todas las pruebas estadísticas se consideraron valores "significativos" aquéllos cuyo nivel de confianza fue del $95 \%(p<0.05)$. 


\section{RESULTADOS}

Con respecto a la situación los encuestados, un 72.7\% habían recibido algún tipo de formación (curso, experto o máster) sobre administración de fármacos en urgencias y un $27.3 \%$ no había recibido ningún tipo de formación sobre la administración de fármacos en urgencias.

Con respecto a su propia autoevaluación, el $72.7 \%$ considera tener unos conocimientos satisfactorios sobre la administración de fármacos y un $27.3 \%$ considera tener un buen conocimiento muy satisfactorio. El $90.9 \%$ conoce la existencia de protocolo sobre la administración de fármacos en su unidad, frente al $9.1 \%$ que no lo conoce. Además, los encuestados poseen una media de formación en este ámbito de 8 años $(D S \pm 8.480)$

Con respecto el nivel de conocimiento sobre la administración de fármacos, tienen una media de aciertos de $6.86(D S \pm 1.457)$, considerándose que un $59.1 \%$ de los encuestados posee conocimientos buenos ( 7 o más aciertos), un $36.4 \%$ tiene conocimientos regulares (5-6 aciertos) y un 4.5\% tiene conocimientos malos o deficientes (menos o igual a 4). Entre los ítems contestados erróneamente, señalar:

Sólo un $76.2 \%$ de los encuestados conoce los efectos adversos de la amiodarona, que son bradicardia e hipotensión (Gráfica 1).

Gráfica 1. Porcentaje de las respuestas a la pregunta de los efectos secundarios más comunes de la amiodarona

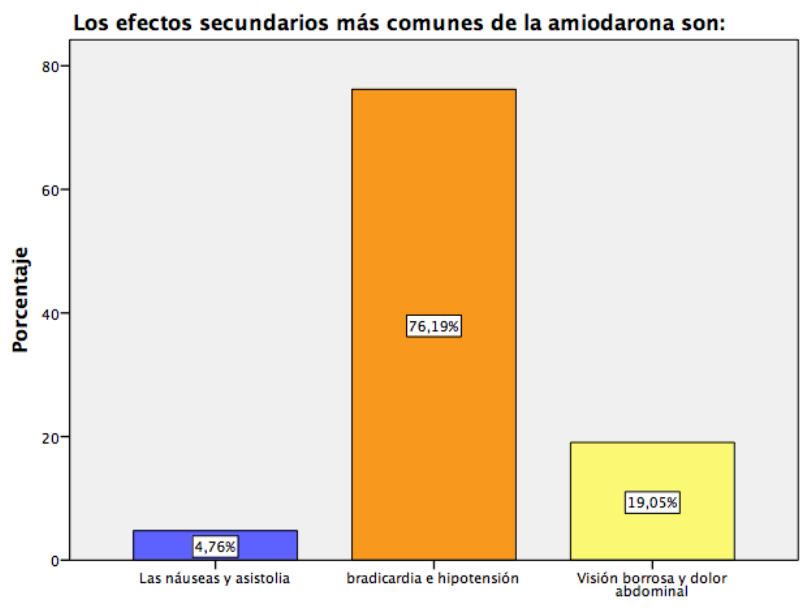

Sólo el $57.1 \%$ de los encuestados conocen que los inhibidores de la enzima convertidora de angiotensina (IECA) se pueden usar en el tratamiento del infarto de miocardio con elevación del ST (Gráfica 2). 
Gráfica 2. Porcentaje de respuestas a la pregunta sobre los inhibidores de la enzima convertidora de angiotensina (IECA)

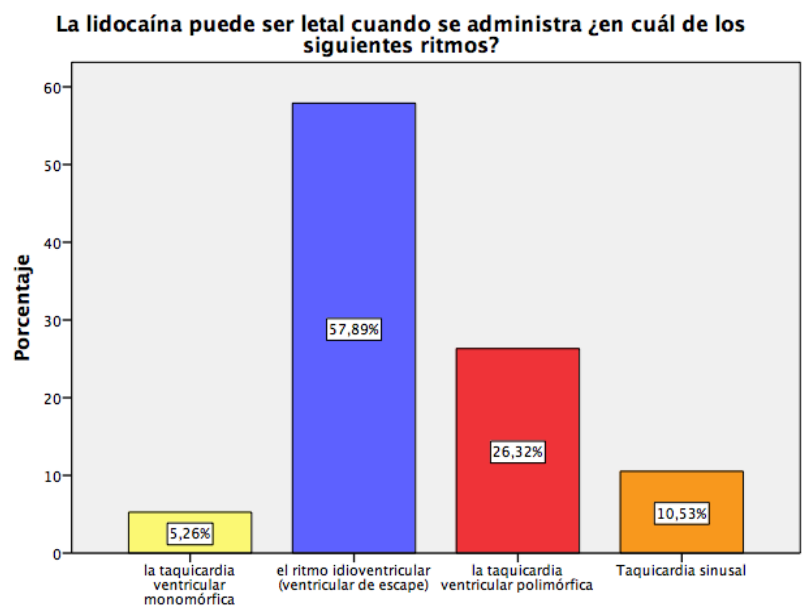

Por último, se comparó las proporciones de las variables categóricas con respecto el nivel de conocimiento de la administración de fármacos, mediante pruebas chi-cuadrado para tablas de contingencia, encontrándose sólo diferencias significativas para un valor de $p=0.018$ al comparar la titulación que posee con el conocimiento de la administración de fármacos, teniendo los médicos mejor conocimiento que los enfermeros (Tabla 1).

Tabla 1. Valor de significación de $p$, al comparar el nivel de conocimientos de administración de medicamentos con la categoría profesional

\begin{tabular}{lccc}
\hline \multicolumn{4}{c}{ Pruebas de chi-cuadrado } \\
\hline & Valor & gl & Sig. asintótica (2 caras) \\
\hline Chi-cuadrado de Pearson & $8.794^{\mathrm{a}}$ & 2 & .012 \\
\hline Razón de verosimilitud & 9.607 & 2 & .008 \\
\hline Asociación lineal por lineal & 8.094 & 1 & .004 \\
\hline $\mathrm{N}$ de casos válidos & 22 & & \\
\hline
\end{tabular}

\section{DISCUSIÓN}

Aunque la mayoría de los encuestados poseen unos conocimientos buenos o regulares sobre la administración de fármacos, según los datos expuestos, el colectivo de enfermeros posee unos conocimientos inferiores al colectivo de médicos en dicha materia. Esto puede ser debido, entre otras causas, a que dichos profesionales poseen una formación en farmacología más extensa en cuanto a horas lectivas y prácticas, además de que para realizar la correcta prescripción deben conocer la ficha técnica del fármaco. 
Teniendo en cuenta que, los enfermeros son el último eslabón en la administración de los medicamentos y es responsabilidad suya su administración, sería conveniente una mayor formación para aumentar los conocimientos en dichos profesionales ya que la formación es el mejor método aumentar los conocimientos y competencias de los profesionales y, de este modo, poder aumentar la calidad de los cuidados (da Silva et al., 2008). La falta de conocimientos sobre la administración de medicamentos en los Servicios de Urgencias puede generar problemas y preocupaciones ya que puede llevar a generar errores, provocando riesgos al paciente atendido, profesional sanitario y a la propia institución como bien podemos comprobar en el estudio realizado por Silva et al. (2009). Esto puede tener implicaciones directas sobre el colectivo de las personas mayores, ya que las personas mayores consumen una gran cantidad de fármacos. De hecho, la media de fármacos consumidos al día de las personas mayores ha aumentado en los últimos años, situándose entre 4.2 y 8 fármacos/persona/día, con un consumo máximo de 18 fármacos distintos al día (Blasco et al., 2004) y son responsables del $70 \%$ del gasto farmacéutico (López de Letona et al., 2005).

Por ello, consideramos necesario la formación, ya que es la única forma de mejorar los conocimientos y competencias de los profesionales para realizar una buena praxis y prestar cuidados de calidad y, sobre todo, seguros (da Silva, da Conceição, y Leite, 2008). Por tanto, al ser las personas mayores las que más fármacos consumen, para que se presten unos cuidados de calidad al colectivo de personas mayores es fundamental que los profesionales sanitarios posean unos conocimientos óptimos sobre medicamentos.

Entre las limitaciones de este estudio destacan que la muestra es insuficiente para extraer conclusiones sólidas y definitivas, por los datos expuestos han de ser considerados como un acercamiento al tema. Entre otras limitaciones, el ser un estudio descriptivo no sirve para medir causalidad, cambios en el tiempo y medir el riesgo de forma directa. Por ello consideramos que sería útil repetir este estudio con un campo muestral más amplio y una mayor dispersión geográfica, que incluya otros hospitales y provincias, para poder extraer resultados más sólidos y definitivos.

Los resultados expuestos son similares a los estudios realizados previamente como el de Machado de Azevedo et al. (2012) en el que aplicó el cuestionario de conocimientos de fármacos a un total de 37 enfermeros en el cual el $32.4 \%$ de ellos acertaron menos de la mitad de los ítems. Otros estudios como el realizado por Mota et al. (2010), cuyo objetivo era evaluar los conocimientos del enfermero de la unidad de cuidados intensivos sobre las recomendaciones para la correcta administración de medicamentos por sonda nasogástrica y nasoentérica, puso de manifiesto que hay una diferencia entre la literatura acerca de los medicamentos administrados por sonda y el conocimiento de los enfermeros sobre el tema. 
En conclusión, aunque el conocimiento de la administración de fármacos es aceptable para el personal sanitario, hay diferencias entre los dos colectivos analizados. Por ello, sería ideal realizar una adecuada formación y reciclaje del personal para conseguir un óptimo conocimiento y, de esta forma, mejorar la atención sanitaria y por consiguiente, reducir los posibles errores en la administración de fármacos. De esta forma podremos prestar unos cuidados de calidad sobre todo a las personas mayores, ya que son un colectivo con alto consumo de medicamentos.

\section{REFERENCIAS}

Abellón, J., Subiela, J.A., Díaz, A.M., Torres, A.M., Aguilón, J.J., y Martínez, M. (2013). Evaluación del Conocimiento sobre Administración de Medicamentos Intravenosos en un Hospital de Segundo Nivel e Implantación de Medidas para su Control. Ciber Revista, 34(4), 2. Disponible en: http://www.enfermeriadeurgencias.com/ciber/ noviembre2013/pagina2.html

Blasco, F., Martínez, J., Pérez, R., Villares, P., Carreño, M.C., y Román, F. (2004). Estudio piloto sobre el consumo de fármacos en ancianos que ingresan en un hospital. Anales de Medicina Interna, 21(2), 69-71.

da Silva, B.K., da Silva, J.S., Gobbo, A.F.F., y Miasso, A.I. (2009). Erros de medicação: condutas e propostas de prevenção na perspectiva da equipe de enfermagem. Revista Eletrônica de Enfermagem, 9(3), 712-723.

da Silva, M.F., da Conceição, F.A., y Leite, M.M.J. (2008). Educação continuada: um levantamento de necessidades da equipe de enfermagem. Mundo da Saúde, 32(1), 47-55.

de Carvalho, V.T., y de Bortoli Cassiani, S.H. (2001). Errores en la administración de medicamentos: análisis de situaciones relatadas por los profesionales de enfermería. Investigación y Educación en Enfermería, 19(2), 26-35.

de Oliveira, R.C., de Camargo, A.E.B., y Cassiani, S.H.D.B. (2005). Estratégias para prevenção de erros de medicação no Setor de Emergência. Revista Brasileira de Enfermagem, 58(4), 399-404.

de Souza, R.B., da Silva, M.J.P., y Nori, A. (2007). Pronto-Socorro: uma visão sobre a interação entre profissionais de enfermagem e pacientes. Revista Gaúcha de Enfermagem, 28(2), 242.

Díaz-Navarlaz, M.T., y Seguí-Gómez, M. (2006). Actitudes, conocimientos y creencias de los profesionales de enfermería sobre errores de medicación. Revista de Calidad Asistencial, 21(1), 6-12.

Elliott, M., y Liu, Y. (2010). The nine rights of medication administration: an overview. British Journal of Nursing, 19(5), 300.

López de Letona, J.M., Patiño, F.B., Jiménez, A., y Villares, P. (2005). El anciano polimedicado: efectos sobre su salud y sobre el sistema sanitario. Información Terapéutica del Sistema Nacional de Salud, 29(6), 152-166.

Low, D.K., y Belcher, J. V. (2002). Reporting medication errors through computerized medication administration. Computers Informatics Nursing, 20(5), 178-183.

Machado de Azevedo, F., Soares, I.M., Rodrigues, C.S., Gomes, P., Tanferri de Brito, T., y Queiroz, A.L. (2012). Administración de medicamentos; conocimiento de los enfermeros del sector de urgencia y emergencia. Murcia: Servicio de Publicaciones de la Universidad de Murcia. 
Ministerio da Saúde, y ANVISA (2013). Protocolo de segurança na prescrição, uso e administração de medicamentos. Brasil: Ministerio da Saúde.

Mota, M.L.S., Barbosa, I.V., Studart, R.M.B., Melo, E.M., Lima, F.E.T., y Mariano, F.A. (2010). Evaluación del conocimiento del enfermero de terapia intensiva sobre administración de medicamentos por sonda nasogástrica y nasoentérica. Revista Latino-Americana de Enfermagem 18(5), 1-8.

O'Leary, D.S. (2000). Accreditation's role in reducing medical errors: Accreditors can provide some leadership, but they cannot do it on their own. BMJ: British Medical Journal, 320(7237), 727.

Pastó-Cardona, L., Masuet-Aumatell, C., Bara-Oliván, B., Castro-Cels, I., Clopes-Estela, A., PaezVives, F., y Codina-Jane, C. (2009). Incident study of medication errors in drug use processes: prescription, transcription, validation, preparation, dispensing, and administering in the hospital environment. Farmacia Hospitalaria (English Edition), 33(5), 257-268.

Rabadán, M., Flores, M.J., Cayuela, J., Cevidades, M.M., Valvuena, R., Ruiz, M.,... y Cervantes, M.D. (2002). Interacciones medicamentosas en la administración de fármacos dentro del proceso de enfermería. Enfermería Global, 1, 1-23.

Recibido: 5 de mayo de 2015 Recepción Modificaciones: 31 de julio de 2015 Aceptado: 3 de agosto de 2015 